that all the crossbridges in a rigor muscle contribute to the stiffness. If this is not so - for example if many are bound in 'slack' states - the calculated duty ratio would be lower and the discrepancy might vanish. If the duty ratio is indeed small, the experiments of Higuchi and Goldman ${ }^{8}$ are consistent with a small working distance. The experiment of Yanagida et al. ${ }^{11}$ remains difficult to explain.

\section{Joe Howard}

Department of Physiology and Biophysics,

University of Washington, Seattle,

Washington 98195, USA

e-mail: johoward@uwashington.edu

1. Howard, J. Nature 389, 561-567 (1997).

2. Finer, J. T., Simmons, R. M. \& Spudich, J. A. Nature $\mathbf{3 6 8}$ 113-119 (1994).

3. Ford, L. E., Huxley, A. F. \& Simmons, R. M. J. Physiol. 269, 441-515 (1977).

4. Huxley, A. F. \& Tideswell, S. J. Musc. Res. Cell Motil. 17, 507-511 (1996).

5. Eisenberg, E., Hill, T. L. \& Chen, Y. Biophys. J. 29, 195-227 (1980)

6. Ford, L. E., Huxley, A. F. \& Simmons, R. M. J. Physiol. 361, 131-150 (1985).

7. Yanagida, T., Arata, T. \& Oosawa, F. Nature 316, 366-369 (1985).

8. Higuchi, H. \& Goldman, Y. E. Nature 352, 352-354 (1991).

9. Huxley, A. F. Prog. Biophys. Biophys. Chem. 7, 255-318 (1957).

10. Lovell, S. J., Knight, P. J. \& Harrington, W. F. Nature 293, 664-666 (1981).

11. Yanagida, T., Arata, T. \& Oosawa, F. Nature 352, 352-354 (1985)

\section{A new classification for $\mathrm{HIV}-1$}

The phenotype of HIV-1 isolates is defined by the cells in which they replicate in vitro, but these phenotypes can change in vivo with profound implications for viral transmission, pathogenesis and disease progression. Here we propose a new classification system based on co-receptor use, providing a more accurate description of viral phenotype than the present imprecise and often misleading classification schemes.

At present, three classification systems are in use. The first defines primary isolates as macrophage (M)-tropic or T-cell-line (T)-tropic. However, this system disguises the fact that all primary isolates replicate in activated, primary CD4+ T-lymphocytes. The second system categorizes isolates as being either syncytium-inducing or nonsyncytium-inducing (NSI) on the basis of whether they form syncytia in MT-2 cells, which express CXCR4 but not CCR5 (ref. 1). However, NSI viruses can readily form syncytia with CCR5-positive cells. The third system defines viruses as either slow/low (SL) or rapid/high (RH) depending on their growth kinetics in culture ${ }^{2}$. These classifications are often used interchangeably, but they are not synonymous.

The identification of some chemokine receptors as having critical roles in the cellular entry of HIV-1 allows us to develop a more precise system for identifying the phenotypic properties of virus strains. A major determinant of HIV-1 tropism (phenotype) lies at the level of virus entry into target cells, which in turn is governed by the expression of co-receptors in conjunction with CD4 (refs 3-5): either CCR5 or CXCR4, or both. CXCR4 use is a defining feature of viruses that form syncytia in Tcell lines; use of CCR5 is a property of NSI, $\mathrm{M}$-tropic viruses; and many T-tropic primary isolates can use both co-receptors ${ }^{3-5}$. A nomenclature based on the co-receptor used would thus provide a precise molecular designation of a given isolate that largely explains its phenotype.

We propose that isolates that use CCR5 but not CXCR4 be termed R5 viruses, that isolates using CXCR4 but not CCR 5 be designated $\mathrm{X} 4$ viruses, and isolates able to use both co-receptors with comparable efficiency be called R5X4. Whether an X4 or R5X4 virus is a cell-line-adapted isolate should also be specified.

Under this system, R5 viruses are the strains most commonly transmitted sexually, consistent with the high resistance of individuals lacking CCR5 to infection ${ }^{6,7}$. After about five years, viruses evolve in about $50 \%$ of patients that are able to use CXCR4, with or without concurrent use of CCR5 (refs 8,9). These viruses would now be called R5X4 and X4 viruses, respectively. Isolates passaged through a permanent $\mathrm{T}$ cell line should be called T-cell line-adapted (TCLA) X4 or R5X4 viruses, and a similar qualifier can be used for viruses adapted to growth on other cells.

This nomenclature takes note of the ability of an isolate to use the major coreceptors, but does not specify whether the isolate can replicate in a particular target cell. The nuances of co-receptor usage in specific contexts are beyond a simple classification system and should be specified by authors if there are perceived ambiguities. That a virus can use a particular co-receptor in transfected cells does not mean that this virus uses the same co-receptor in a more physiological context. The efficiency with which different co-receptors are used by some strains is likely to vary between assay systems; again, authors should clearly explain the limitations of their results, and the significance of extremely low efficiency co-receptor usage should not be over-interpreted. This classification system can also be expanded to take note of other co-receptors if their use by an isolate proves to be a major determinant of tropism; for example, whether isolates use CCR3 and/or CCR5 to enter microglia could define them as R3, R5 or R3-R5 isolates ${ }^{10}$.

Our classification system is uncomplicated, is intuitive to those familiar with the usage of the CCR5 and CXCR4 co-receptors by HIV-1, and removes the inaccuracies and confusion associated with the present systems. It is flexible and open to expansion to accommodate emerging knowledge of co-receptor usage, and can encompass HIV-2 and SIV strains, which also use CCR5 and other co-receptors for entry $^{3-5}$.

A record of co-receptor use by particular HIV-1, HIV-2 and SIV strains will be maintained by the Los Alamos National Laboratory Sequence Database, together with an expanded version of this article.

\section{E. A. Berger}

Laboratory of Viral Diseases, NIAID,

National Institutes of Health, Building 4,

Room 236, Bethesda, Maryland 20892, USA

\section{R. W. Doms}

Department of Pathology and Laboratory Medicine, University of Pennsylvania, Philadelphia,

Pennsylvania 19104, USA

E.-M. Fenyö

EC Concerted Action on HIV Variation,

Microbiology and Tumorbiology Center,

Karolinska Institute, 171 77, Stockholm, Sweden

B. T. M. Korber

Theoretical Biology and Biophysics, Los Alamos

National Laboratory, Los Alamos,

New Mexico 87545, USA

\section{R. Littman}

Howard Hughes Medical Institute,

New York University Medical Center, New York,

New York 10016, USA

\section{J. P. Moore}

The Aaron Diamond AIDS Research Center,

The Rockefeller University, 455 First Avenue,

New York, New York 10016, USA

\section{Q. J. Sattentau}

Centre d'Immunologie de Marseille-Luminy, Case 906, 13288 Marseille, France

\section{H. Schuitemaker}

Department of Clinical Viro Immunology, Central Laboratory of the Netherlands Red Cross Blood Transfusion Service and Laboratory for Experimental and Clinical Immunology,

University of Amsterdam, Amsterdam,

The Netherlands

\section{J. Sodroski}

Department of Cancer Immunology/AIDS, Dana-Farber Cancer Institute, 44 Binney Street, Boston, Massachusetts 02115, USA

\section{R. A. Weiss}

Chester Beatty Laboratories,

The Institute of Cancer Research, 237 Fulham

Road,

London SW3 6JB, UK

1. Schuitemaker, H. et al. J. Virol. 66, 1354-1360 (1992).

2. Fenyö, E. et al. J. Virol. 62, 4414-4419 (1988).

3. Berger, E. A. AIDS 11 (suppl. A), S3-S16 (1997).

4. Moore, J. P., Trkola, A. \& Dragic, T. Curr. Opin. Immunol. 9 , 551-562 (1997).

5. Doms, R. W. \& Peiper, S. C. (1997) Virology 235, 179-190.

6. Liu, R. et al. Cell 86, 367-377 (1996).

7. Samson, M. et al. Nature 382, 722-725 (1996).

8. Richman, D. D. \& Bozzette, S. A. J. Infect. Dis. 169, 968-974 (1994).

9. Connor, R. I. \& Ho, D. D. J. Virol. 68, 4400-4408 (1994). 10. He, J. et al. Nature 385, 645-649 (1997). 